\title{
Comparison of Phenolic Compounds and Antioxidant Activities of Raw and Cooked Turkish Artichoke Cultivars
}

\section{OPEN ACCESS}

Edited by:

Patricia Morales,

Complutense University of

Madrid, Spain

Reviewed by:

Irda Fidrianny,

Bandung Institute of

Technology, Indonesia

Kunchit Judprasong,

Mahidol University, Thailand

${ }^{*}$ Correspondence:

Didem Saloglu

saloglu@itu.edu.tr

orcid.org/0000-0003-0503-056X

Specialty section:

This article was submitted to Nutrition and Sustainable Diets,

a section of the journal

Frontiers in Sustainable Food Systems

Received: 19 August 2021

Accepted: 01 October 2021

Published: 18 November 2021

Citation:

Kayahan S and Saloglu D (2021) Comparison of Phenolic Compounds and Antioxidant Activities of Raw and

Cooked Turkish Artichoke Cultivars.

Front. Sustain. Food Syst. 5:761145.

doi: 10.3389/fsufs. 2021.761145

\section{Seda Kayahan ${ }^{1}$ and Didem Saloglu ${ }^{2 *}$ \\ ${ }^{1}$ Department of Chemical Engineering, Institute of Graduate Studies, Yalova University, Yalova, Turkey, ${ }^{2}$ Disaster and Emergency Management Department, Disaster Management Institute, Istanbul Technical University, Istanbul, Turkey}

The objective of this work was to determine the total phenolic compounds and antioxidants in raw and cooked Sakiz and Bayrampasa variety artichokes in parts such as inner bracts, stems, receptacles, and outer bracts. The artichokes were cooked by boiling, microwaving, and baking methods, and total phenolic compounds and antioxidants of cooked artichokes were evaluated. While TPC (total phenolic content), DPPH (2,2-diphenyl-1-picryl-hydrazine), and CUPRAC values for the leaves of raw Bayrampasa artichoke were found to be $686 \mathrm{mg}$ gallic acid equivalent (GAE)/100 g, $478 \mathrm{mg}$ TE/100 g, and 4,875 mg TE/100 g, respectively, TPC, DPPH, and CUPRAC values for stems of Sakiz artichoke were determined to be $1,579 \mathrm{mg}$ GAE/100 $\mathrm{g}$, $1,259 \mathrm{mg}$ TE/100 $\mathrm{g}$, and 3,575 mg TE/100 g. A significant increase in the content of TPC, DPPH, and CUPRAC values was observed for all cooking applications of both artichokes. DPPH and CUPRAC values increased by 11 and 43 times and 17 and 6.7 times after baking of Sakiz and microwave cooking of Bayrampasa, respectively. Chlorogenic acid, cynarine, and cynaroside content of both artichokes had an increment after all cooking applications. Chlorogenic acid content was improved 29 and 58 times after baking of Sakiz and microwave cooking of Bayrampasa, respectively.

Keywords: artichoke, cooking, phenolics, antioxidant, activity

\section{INTRODUCTION}

Artichoke (Cynara scolymus L.) is a perennial plant variety of the Asteraceae family, traditionally grown in the Mediterranean region, which is an important multipurpose plant used in salads, soups, and meals. In addition, it is an important source of raw materials in industries related to liquor, paint, pharmacy, and animal feed. The head and the other parts of the artichoke are consumed fresh, canned, or frozen (Lattanzio et al., 2009). Many studies found that artichoke has cholesterol-lowering, antioxidant, anticancer, diuretic, anti-inflammatory, antifungal, and antibacterial properties (Christaki et al., 2012). It is used as a functional food for intestinal infections, constipation, obesity, colorectal cancer, and liver diseases because of its polyphenolic compounds, dietary fibers, and inulin (Angelov et al., 2015).

The highest varieties of artichoke are produced in Italy, followed by Egypt and Spain. Turkey ranks 10th in world with 39,000 tons of annual artichoke production. In Turkey, domestic artichoke genotypes are produced to a large extent. Whereas the "Bayrampasa" genotype, which has late characteristics, is produced in the Marmara region, the "Sakiz" artichoke, which is an early genotype, is produced in the Aegean region. 
The polyphenolic content of artichoke depends on genetic and environmental factors, but it was stated that the phenolics in artichoke are higher than many vegetables. Both the edible part of the artichoke and its by-products mainly contain caffeoylquinic acids, di-caffeoylquinic acids, filaments, and dietary fibers (Pandino et al., 2013). The important antioxidant compounds in artichoke include cynarine, chlorogenic acid (5-O-caffeoylquinic acid), 1,5-di-O-caffeoylquinic acid, 3,4-di-O-caffeoylquinic acid, 3,5-di-O-caffeoylquinic acids, luteolin, and apigenin glycosides and their derivatives (Christaki et al., 2012).

The antioxidant capacity of cooked artichokes was reported. Different cooking methods, such as boiling, microwaving, steaming, grilling, and frying, were applied to artichoke, and the phytochemical profiles and antioxidant activities increased significantly.

In order to improve the functional activities of vegetables, the optimum cooking method should be determined to keep the antioxidant capacity and radical scavenging activity at the highest levels. Comprehensive data about the comparison of raw and cooked Sakiz and Bayrampasa artichokes are not available in the literature; thus, the aim of this study was to compare the phenolic compounds and antioxidant activities of two local genotype artichokes, both raw and cooked Sakiz and Bayrampasa varieties. Total phenolic compounds, antioxidant activities, and amounts of phenolic substances in raw and cooked Sakiz and Bayrampasa artichokes were determined.

\section{MATERIALS AND METHODS}

\section{Artichoke Sample Preparation}

C. scolymus L. cv. Sakiz and Bayrampasa artichokes were collected from Izmir and Bursa in Turkey, respectively. Artichokes were transported under refrigerated conditions within maximum $1 \mathrm{~h}$ from harvesting and stored at $4^{\circ} \mathrm{C}$ until use.

Moisture content was determined following the guidelines of the official TS 1129-ISO 1026 method (Turkish Standard, 1998). For each sample, $\sim 2 \mathrm{~g}$ was placed on pre-weighed aluminum pans and weighed. The pans were kept in vacuum oven at $70^{\circ} \mathrm{C}$ until the equilibrium moisture content was reached during $6 \mathrm{~h}$. All samples were analyzed in triplicate, and average values were calculated.

\section{Chemicals}

Gallic acid, Trolox [( \pm -6-hydroxy-2,5,7,8tetramethylchromane-2-carboxylic acid], DPPH (2,2-diphenyl1-picryl-hydrazine), Folin-Ciocâlteu's phenol reagent, neocuproine, and sodium carbonate were purchased from Sigma-Aldrich, France. Methanol was purchased from JT Baker Chemicals, Poland. Ethanol, ammonium acetate, and copper (II) chloride dihydrate $\left(\mathrm{CuCl}_{2} \cdot 2 \mathrm{H}_{2} \mathrm{O}\right)$ was purchased from Merck, Germany. The phenolic standards were obtained as follows: chlorogenic acid, cynarine (1,3-dicaffeoylquinic acid), cynaroside (luteolin 7-glucoside), luteolin, syringin, and rhoifolin (apigenin7-O- neohesperidoside) were purchased from Sigma-Aldrich, Germany. All reagents were of analytical grade.

\section{Cooking Treatments}

Boiling, microwave cooking, and baking were used as cooking methods (Ferracane et al., 2008). Sakiz and Bayrampasa artichokes were immediately peeled. Receptacles (hearts) of the artichokes were kept in 3.0\% (wt/vol) NaCl and 5\% (wt/vol) citric acid solution at $20^{\circ} \mathrm{C}$ for $8 \mathrm{~h}$ for pretreatment. Then, the cooking processes were carried out in triplicate.

\section{Boiling}

The receptacles (heart) of Sakiz and Bayrampasa artichokes were added to boiling fresh water in a covered stainless-steel pot (food/water, 1:5) and cooked on a moderate flame in triplicate. For each cooking trial, five parts were boiled; cooked samples were drained after $18 \mathrm{~min}$ (Rinaldi et al., 2020). After cooling, samples were stored at $-18^{\circ} \mathrm{C}$ until analysis.

\section{Microwave Cooking}

The receptacles (heart) of Sakiz and Bayrampasa artichokes were placed in 1-L beakers (food/water, 1:1) and cooked at $800 \mathrm{~W}$ in a microwave oven for $12.5 \mathrm{~min}$ for both artichokes.

\section{Baking}

The receptacles (heart) of Sakiz and Bayrampasa artichokes were placed in a ceramic dish in a $170^{\circ} \mathrm{C}$ preheated oven during $70 \mathrm{~min}$ (Jiménez-Monreal et al., 2009).

\section{Extraction Procedure}

One gram of cooked artichoke samples and $50 \mathrm{~mL}$ of pure methanol were placed in a water bath at $70^{\circ} \mathrm{C}$ for $2 \mathrm{~h}$. After extraction, the extraction sample was centrifuged $(8,000 \mathrm{~g})$. The extracts were stored at $-20^{\circ} \mathrm{C}$ until analysis (Stumpf et al., 2019).

\section{Total Phenolic Content}

The total phenolic contents (TPCs) of extract phases were assessed spectrophotometrically using the Folin-Ciocâlteu procedure (Kayahan and Saloglu, 2020). TPC values were calculated as milligram gallic acid equivalents (GAE) per $100 \mathrm{~g}$ of dry weight. All TPC analyses were performed four times.

\section{DPPH Antioxidant Activity Determinations}

The DPPH antioxidant activities of extract phases were examined according to Kayahan and Saloglu (2020). DPPH values were calculated as milligram Trolox equivalent per $100 \mathrm{~g}$ of dry weight. All DPPH analyses were performed four times.

\section{Copper Reducing Antioxidant Capacity}

The CUPRAC antioxidant capacities of extract phases were assessed spectrophotometrically as previously reported (Apak et al., 2004). CUPRAC antioxidant capacity values were calculated as milligram Trolox equivalent per $100 \mathrm{~g}$ of dry weight. All CUPRAC analyses were performed four times.

\section{Quantitative Determination of Phenolic Compounds in Artichoke Cultivars by HPLC}

Phenolic compounds were analyzed using a series 1100 HPLC (Hewlett-Packard, Waldbronn, Germany), equipped with a 


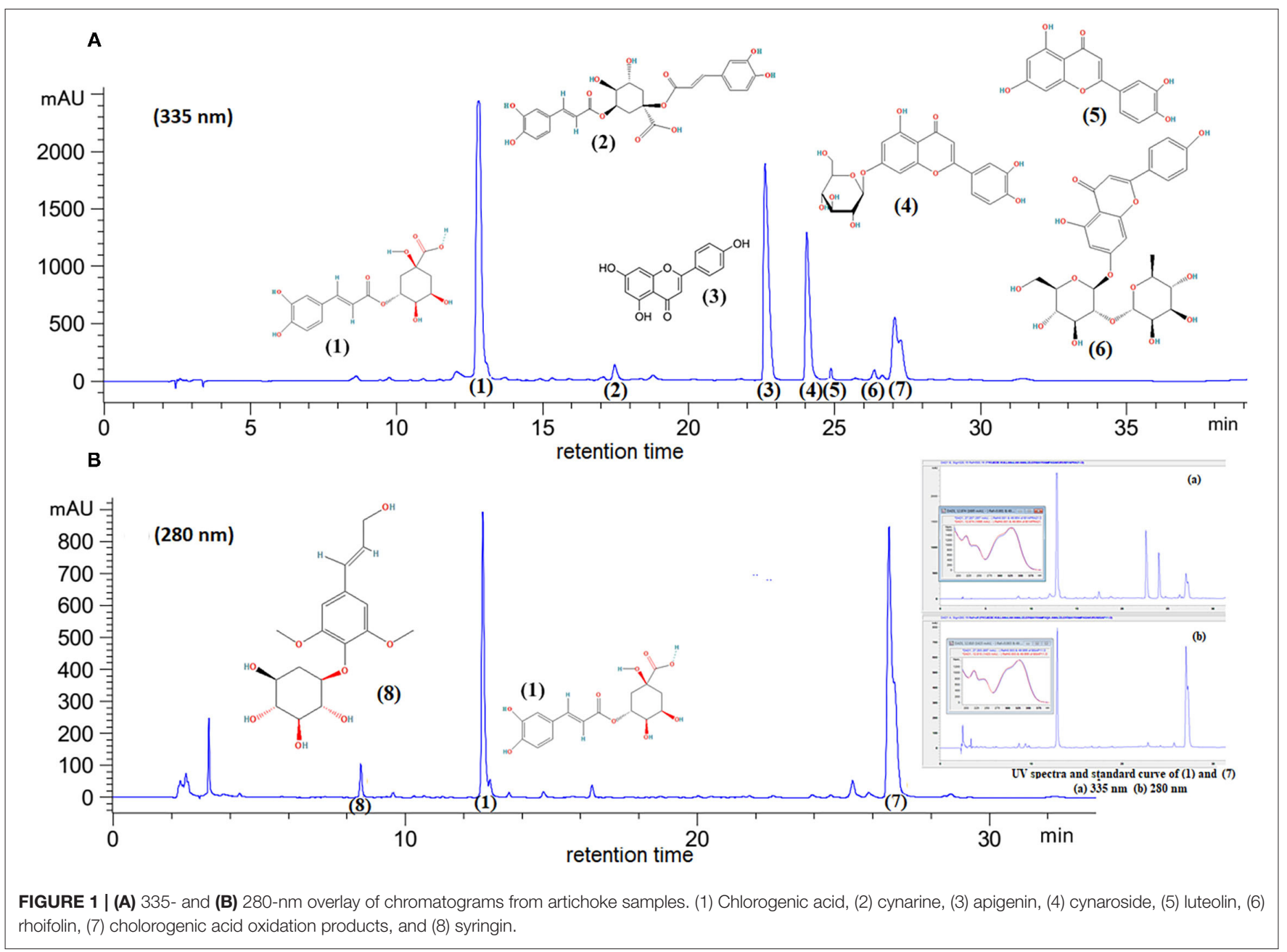

G1315A diode array detection system. Separation was conducted using a chromatography column with 150-mm length, 4.6$\mathrm{mm}$ width, and particle size $2.6 \mu \mathrm{m}$. The phenolic standard solutions and mixtures were injected into the system using an autoinjector. Different isocratic and gradient mobile phases were tested at different flow rates and column temperatures in order to find a suitable separation method for the standards. The gradient method was chosen following series of preliminary uses with a mixture of phosphoric acid in water (mobile phase A) and acetonitrile (mobile phase B). The total run time was $50 \mathrm{~min}$. The gradient program was as follows: initial $6 \% \mathrm{~B}$, linear gradient to $30 \% \mathrm{~B}$ in 20 min hold at $30 \%$ for $5 \mathrm{~min}$. The post running time was $10 \mathrm{~min}$. Following the analysis of the UV-Vis spectra of the individual phenolic standards, 280- and 335-nm wavelengths were chosen for investigation of chlorogenic acid, cynarine, apigenin, cynaroside, luteolin, rhoifolin, and syringin using the HPLC-DAD (Figure 1).

In Figure 1, the numbers refer to the major phenolic compounds identified: (1) chlorogenic acid, (2) cynarine, (3) apigenin, (4) cynaroside, (5) luteolin, (6) rhoifolin, (7) cholorogenic acid oxidation products, and (8) syringin.
Chlorogenic acid (5'-caffeoylquinic acid) possesses two adjacent hydroxyl groups, which oxidize to cholorogenic acid quinone in the presence of oxygen. It can be safely stated that oxidation products of chlorogenic acid represented by the peak named (7) was hydroxyl derivatives of chlorogenic acid (Luo et al., 2016).

\section{Statistical Analyses and Experimental Design}

The data are presented as mean \pm the standard deviation. Oneway analysis of variance with Tukey test was performed by using Statgraphics software in order to determine differences in TPC, DPPH, CUPRAC, and phenolic compound values of the receptacle (heart), inner bracts, outer bracts, leaves, and stem of artichokes. Differences were considered significant at $p<0.05$ (Kayahan and Saloglu, 2020).

\section{RESULTS AND DISCUSSION}

\section{Moisture Content}

The moisture content of the artichoke parts is reported in Table 1. The moisture contents of leaves, stem, outer bracts, receptacle, 
and inner bracts of raw Sakiz artichoke were recorded to be $85,91,89,83$, and $84 \%$, respectively. In addition, the moisture content of Bayrampasa parts were recorded to be $80 \%$ in leaves, $72 \%$ in stem, $74 \%$ in outer bracts, $80 \%$ in receptacle, and $74 \%$ in inner bracts. After all cooking processes, Sakiz had lower moisture content compared to Bayrampasa.

\section{TPC, DPPH, and CUPRAC Values and Phenolic Compounds of Raw Artichokes}

TPC of both artichoke genotypes was expressed on a dry weight basis. TPC of Bayrampasa artichoke is reported in Figure 2A. Inner bracts, stems, receptacles, and outer bracts of raw Bayrampasa artichoke exhibited significantly lower amounts of total phenolic compounds compared with leaves, whereas the TPC values of receptacle, inner bracts, outer bracts, leaves, and stem of raw Bayrampasa were recorded to be 212, 139, 347, 686, and $228 \mathrm{mg} \mathrm{GAE} / 100 \mathrm{~g}$, respectively. All parts of raw Sakiz artichoke had higher amounts of TPC than that of Bayrampasa. TPC values of receptacle, inner bracts, outer bracts, leaves, and stems were determined to be $844,1,070,869,1,384$, and 1,579 mg GAE/100 g, respectively (Figure 2B).

DPPH spectrophotometric assay results had a similar trend to those recorded for TPC results of DPPH values of receptacle, inner bracts, outer bracts, leaves, and stem of Bayrampasa, which were recorded to be $298,112,201,478$, and $215 \mathrm{mg} \mathrm{TE} / 100 \mathrm{~g}$,

TABLE 1 | Moisture content of raw and cooked artichokes.

Sakiz Bayrampasa

Moisture content (\%)

Raw leaves

Raw stem

85

80

Raw outer bracts

91

72

Raw receptacle

89

74

Raw inner bracts

83

80

Boiling

Microwave cooking

Baking respectively (Figure 2A). Leaves and stems of Sakiz artichoke have higher DPPH amounts than the others. DPPH values of receptacle, inner bracts, outer bracts, leaves, and stem of Sakiz artichoke were found to be $628,708,100,1,015$, and $1,259 \mathrm{mg}$ TE/100 g, respectively (Figure 2B).

The leaves of raw Bayrampasa artichoke had significantly higher amount of CUPRAC compared with the other parts. CUPRAC values of receptacle, inner bracts, outer bracts, leaves, and stem of Bayrampasa artichoke were determined to be 2,183, $808,654,4,875$, and 1,366 mg TE/100 g, respectively (Figure 2A). Also, the change in CUPRAC values was similar to TPC values for all parts of raw Sakiz artichoke. The lowest value of CUPRAC was determined in the outer bracts. Although the CUPRAC value was the lowest in the outer bracts, it was found at highest value in the stem of raw Sakiz artichoke. The highest and lowest values were found to be 3,575 and $414 \mathrm{mg} \mathrm{TE} / 100 \mathrm{~g}$ in the stem and outer bracts, respectively (Figure $2 \mathbf{B}$ ).

Significant correlations were observed between TPC, DPPH, and CUPRAC data $\left(R^{2}>0.95\right)$, confirming the high levels of polyphenolic compounds in artichokes, which were responsible for their total antioxidant capacity.

Falleh et al. indicated that artichokes were widely consumed in Tunisia (Cynara cardunculus) and examined phenolic contents and antioxidant activities of artichoke. The highest phenolic compounds and antioxidant activity were determined in the leaves, whereas the lowest phenolic compounds were found in the seed part. All artichoke parts were identified to have high polyphenol content of 7.5 and $15 \mathrm{mg}$ GAE/g. The polyphenol contents of parts of five different artichokes were examined, and different artichoke parts were found to have different polyphenol contents. The highest phenol content was found in the inner bract and leaves, whereas the lowest was found in the outer bract (Falleh et al., 2008).

The unclear relationship between antioxidant activity and total phenolics was explained in several ways: the total phenolic fraction does not incorporate all the antioxidants, and synergistic interactions between the antioxidants in the mixture make the antioxidant activity dependent not only on the concentration, but also on the structure and the nature of the antioxidants. The phenolic content of a plant depends on
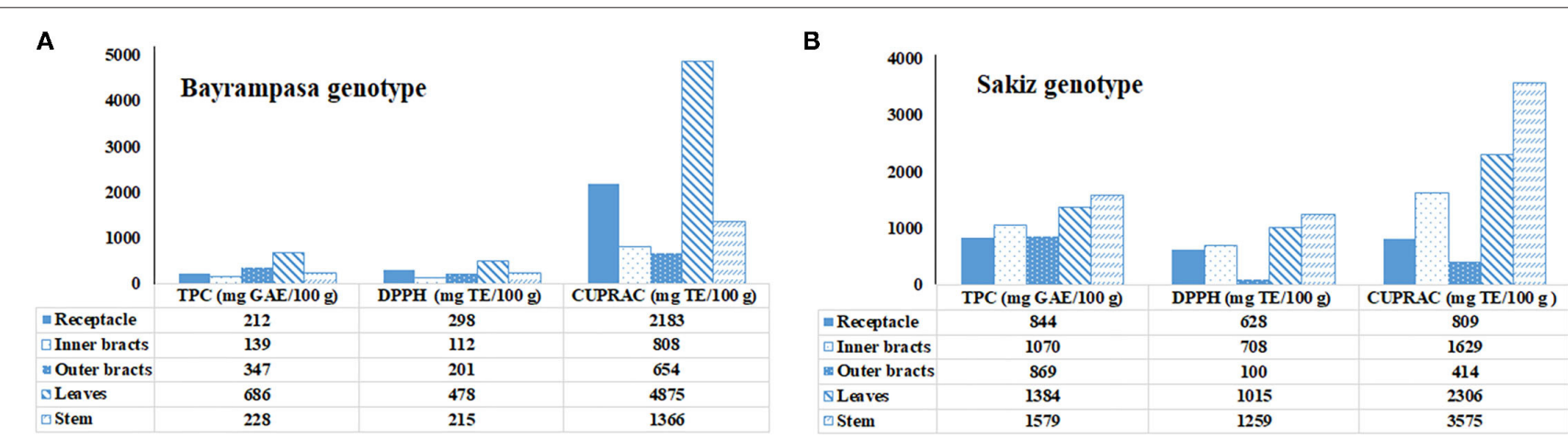

FIGURE 2 | TPC, DPPH, and CUPRAC values of different parts of raw Turkish artichokes (A: Bayrampasa genotype and B: Sakiz genotype) 
TABLE 2 | Phenolic substance content of different parts of raw artichokes.

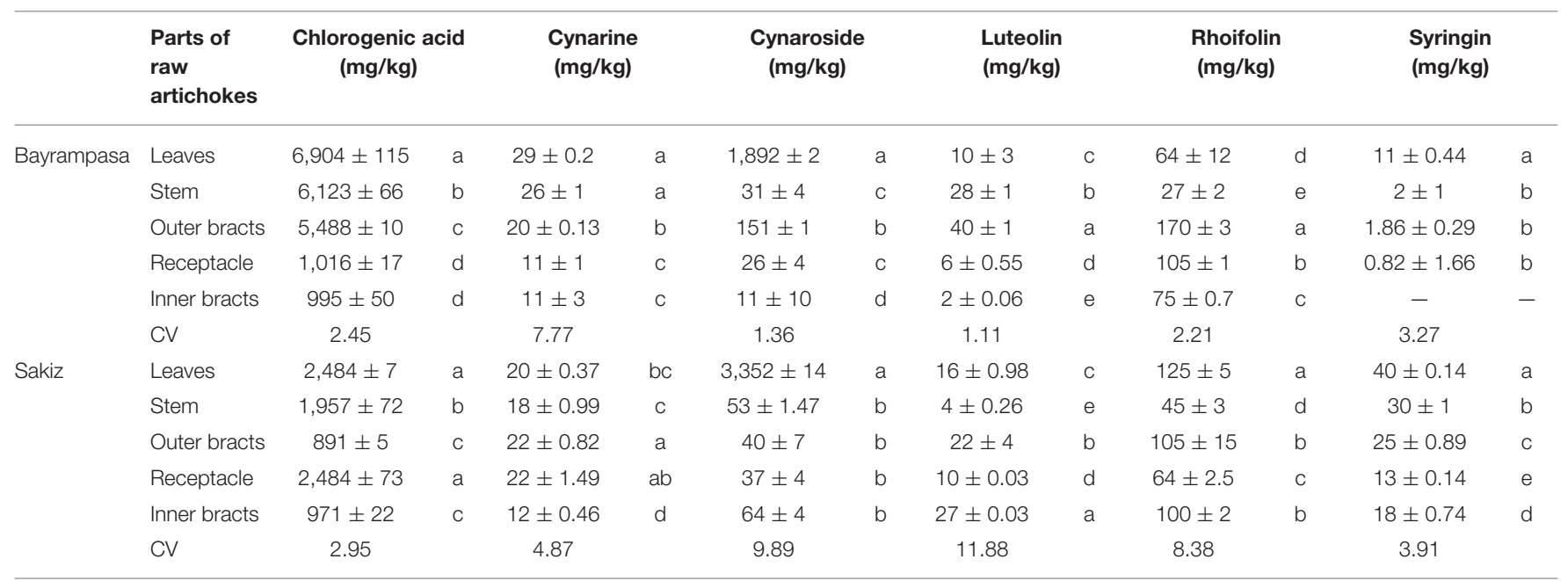

$a, b, c, d$, and e represent interaction of the factors.

a number of genetic, environmental, processing, and storage factors (Fratianni et al., 2007).

Important phenolic compounds for sections belonging to the raw Sakiz and Bayrampasa artichokes were studied. The leaf extracts of Bayrampasa artichoke showed significantly higher content of chlorogenic acid and cynarine compared to leaves of Sakiz (Table 2). The chlorogenic acid in the leaves of Bayrampasa artichoke was determined to have the highest value $(6,904$ $\mathrm{mg} / \mathrm{kg}$ ). The receptacles of raw Bayrampasa and Sakiz artichokes contain 1,016 and 2,484 mg/kg chlorogenic acid. The highest chlorogenic acid was found to be 6,904 and $2,484 \mathrm{mg} / \mathrm{kg}$ in the leaf part of both artichoke types. The chlorogenic acid in stem, outer bracts, receptacle, and inner bracts of Bayrampasa and Sakiz was calculated as 6,123 and 1,957, 5,488 and 891, 1,016 and 2,484, and 995 and $971 \mathrm{mg} / \mathrm{kg}$, respectively. The high chlorogenic acid in the artichokes can be explained by its central role as a substrate for many biochemical reactions that produce several phenolic acids highly represented in artichokes. The leaves have higher phenolic content than the other parts, and also chlorogenic acid contents were higher than the others for both genotypes. Also, cynaroside and rhoifolin were determined to be 3,352 and 125 and 1,892 and $64 \mathrm{mg} / \mathrm{kg}$ in the leaves of Sakiz and Bayrampasa artichokes, respectively. The rhoifolin in stem, outer bracts, receptacle, and inner bracts of Bayrampasa and Sakiz were calculated as 27 and 45, 170 and 105, 105 and 64, and 75 and $100 \mathrm{mg} / \mathrm{kg}$, respectively. Luteolin and syringin contents were the lowest for both Sakiz and Bayrampasa. The luteolin in leaves, stem, outer bracts, receptacle, and inner bracts of Bayrampasa and Sakiz were calculated as 10 and 16, 28 and 4, 40 and 22, 6 and 10 , and 2 and $27 \mathrm{mg} / \mathrm{kg}$, respectively. Syringin was detected to be the lowest value $(2 \mathrm{mg} / \mathrm{kg})$ in the stem of Bayrampasa.

In the present study, it was determined that the phenolic content of the non-edible parts of both artichokes was higher than the other parts. The highest cynarine content was found in the leaves and stems of Bayrampasa, whereas it was found the highest in the outer bracts and receptacle of Sakiz artichoke.

\section{TPC, DPPH, and CUPRAC Values and Phenolic Content of Cooked Artichokes With Different Cooking Methods}

Artichokes are an important multipurpose vegetable used in salads, soups, and dishes that are important in the Mediterranean diet (Falleh et al., 2008). Nowadays, boiled, microwaved, baked, steamed, grilled, fried, and cooked vegetables receive great interest for their phytochemical profiles and antioxidant activities. In order to improve the functional activities of vegetables, it is important to determine the optimal cooking method, which causes retention of antioxidant capacity and radical scavenging activity at the highest level. Therefore, it is important to know what happens to the antioxidant activity or free radical capacity during common domestic cooking methods. Total phenolic compounds and antioxidant activities were investigated via three different cooking methods for Bayrampasa and Sakiz artichokes, which are most intensively eaten in Turkey.

For the cooking methods, the highest total phenolic compounds were found to be present in the microwave method, whereas the lowest phenolic compounds were determined in the baking method for Bayrampasa genotype. The highest TPC was determined to be $2,478 \mathrm{mg}$ GAE/ $100 \mathrm{~g}$ during microwave and 2,447 mg GAE/100 g during boiling cooking for Bayrampasa. The lowest total phenolic compounds were found after baking at 1,524 mg GAE/100 g (Table 3). Whereas DPPH antioxidant activity was prominent in microwave cooking, baking is the method with the lowest antioxidant activity. The highest values for antioxidant activity of DPPH and CUPRAC were 12,815 and 14,600 $\mathrm{mg} \mathrm{TE} / 100 \mathrm{~g}$, respectively, after microwave cooking for Bayrampasa, whereas DPPH and CUPRAC antioxidant activities were 6,146 and $7,767 \mathrm{mg} \mathrm{TE} / 100$, respectively, for baking. In addition, DPPH and CUPRAC antioxidant activities were 10,374 and $9,548 \mathrm{mg} \mathrm{TE} / 100$, respectively, after boiling for Bayrampasa genotype. The lowest values for DPPH and CUPRAC antioxidant activity were determined after baking and boiling methods, respectively. 
TABLE 3 | TPC, DPPH, and CUPRAC values of raw and cooked artichokes.

\begin{tabular}{|c|c|c|c|c|c|c|c|c|c|c|}
\hline \multirow{3}{*}{$\begin{array}{l}\text { Total phenolic content } \\
\text { (mg GAE/100 g) }\end{array}$} & \multirow[b]{2}{*}{ Sakiz } & \multirow{2}{*}{$\begin{array}{c}\text { Raw (receptacle) } \\
844\end{array}$} & \multicolumn{2}{|l|}{ Boiling } & \multicolumn{2}{|c|}{ Microwave cooking } & \multicolumn{2}{|l|}{ Baking } & \multirow{2}{*}{$\begin{array}{c}\text { Mean } \\
1,739 \mathrm{~B}\end{array}$} & \multirow{2}{*}{$\frac{\text { CV }}{5.83}$} \\
\hline & & & $1,836 \pm 14$ & $\mathrm{~b}$ & $1,511 \pm 12$ & c & $1,870 \pm 14$ & b & & \\
\hline & Bayrampasa & 212 & $2,447 \pm 17$ & a & $2,478 \pm 78$ & a & $1,524 \pm 8$ & c & $2,149 \mathrm{~A}$ & \\
\hline & Mean & & $2,142 \mathrm{~A}$ & & $1,995 \mathrm{~B}$ & & $1,697 \mathrm{C}$ & & & \\
\hline \multirow{2}{*}{$\begin{array}{l}\text { DPPH antioxidant activity } \\
\text { (mg TE/100 g) }\end{array}$} & Sakiz & 628 & $5,724 \pm 72$ & d & $4,790 \pm 27$ & e & $7,093 \pm 27$ & c & $58.69 \mathrm{~B}$ & 7.63 \\
\hline & Bayrampasa & 298 & $10,374 \pm 16$ & $\mathrm{~b}$ & $12,815 \pm 114$ & $\mathrm{a}$ & $6,146 \pm 59$ & $d$ & $9,779 \mathrm{~A}$ & \\
\hline (mg TE/100 g) & Bayrampasa & 2,183 & $9,548 \pm 54$ & $\mathrm{~b}$ & $14,600 \pm 166$ & a & $7,767 \pm 113$ & c & 10,639 & \\
\hline & Mean & & $9,792 B$ & & $12,307 A$ & & 10,577B & & & \\
\hline
\end{tabular}

$a, b, c$, and $d$ represent interaction of the factors and $A, B$, and $C$ represent one factor.

TABLE 4 | Phenolic substance content of raw and cooked artichokes.

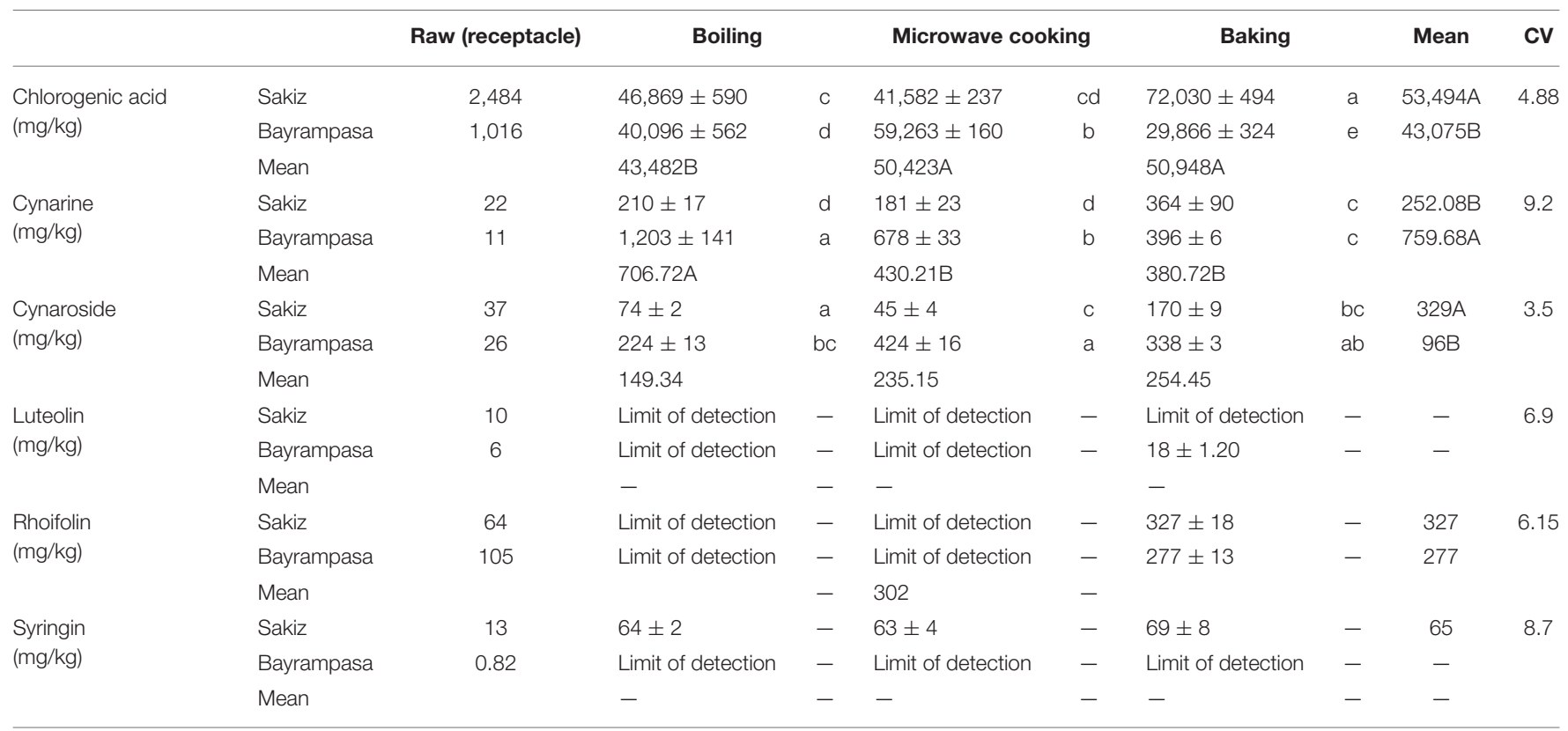

$a, b, c$, and $d$ represent interaction of the factors and $A$ and $B$ represent one factor.

The highest TPC was determined to be $1,870 \mathrm{mg}$ GAE/100 $\mathrm{g}$ during baking cooking for Sakiz genotype. The lowest total phenolic compounds were found after microwave at $1,511 \mathrm{mg}$ $\mathrm{GAE} / 100 \mathrm{~g}$. The highest values for antioxidant activity of DPPH and CUPRAC were 7,093 and 13,386 mg TE/100 g, respectively, after baking cooking for Sakiz genotype. In addition, the lowest values for DPPH and CUPRAC antioxidant activity were determined after microwave cooking. DPPH and CUPRAC antioxidant activity values were found to be 4,790 and $10,015 \mathrm{mg}$ $\mathrm{TE} / 100 \mathrm{~g}$, respectively for Sakiz. The most appropriate cooking method can be said to be baking for Sakiz artichoke, whereas the highest loss in nutritional value was observed in microwave cooking. Also, for Bayrampasa genotype, the lowest nutrient loss was observed for the microwave cooking application.

These phenolic compounds cannot completely explain the differences in the antioxidant capacity observed in cooked artichokes, which could be also attributed to the conversion of polyphenols into active antioxidant species that synergistically determine a very high antioxidant capacity. In addition, the higher phenolic content after thermal treatment can be due to acting on cell wall membranes, yielding a softening and disrupting effect that promoted the release of internal compounds. Also, the presence of water in the cooking process can extract a percentage of the released compounds.

The effects of boiling, microwaving, and baking Sakiz and Bayrampasa artichokes on polyphenolic contents were analyzed using HPLC DAD (Table 4). A significant increase in the content of chlorogenic acid, cynarine, and cynaroside was observed for all cooking applications of both artichokes. Chlorogenic acid had the highest value in baking cooking applications, and it was detected to be $72,030 \mathrm{mg} / \mathrm{kg}$ in Sakiz artichoke, whereas it was determined to be 59,263 $\mathrm{mg} / \mathrm{kg}$ after microwave cooking in Bayrampasa. Cynarine had the highest value in Bayrampasa artichoke for all cooking 
methods. The highest cynarine content was detected with the boiling method for Bayrampasa $(1,203 \mathrm{mg} / \mathrm{kg})$. Also, the highest cynarine content was determined to be 364 $\mathrm{mg} / \mathrm{kg}$ after baking method for Sakiz artichoke, whereas the lowest cynarine content was $181 \mathrm{mg} / \mathrm{kg}$ in microwave cooking application (Table 4).

Increasing contents of caffeoylquinic acid isomers were determined during all cooking methods, caused by the trans-esterification of 5-O-caffeoylquinic and 1,5-di-Ocaffeoylquinicacids at high temperatures. In addition, the bleaching process before the artichoke cooking process was shown to affect phenolic content and antioxidant activities. This increase can be attributed to the inactivation of the polyphenol oxidase enzyme. Cynaroside was found to be 224 and 74, 424 and 45 , and 338 and $170 \mathrm{mg} / \mathrm{kg}$ after boiling, microwave, and baking cooking for Bayrampasa and Sakiz artichokes, respectively.

Luteolin, rhoifolin, and syringin were not detected effectively in both artichokes. Luteolin was found to be $18 \mathrm{mg} / \mathrm{kg}$, whereas rhoifolin was determined to be $277 \mathrm{mg} / \mathrm{kg}$ after baking cooking of Bayrampasa. In addition, rhoifolin and syringin were found to be 327 and $69 \mathrm{mg} / \mathrm{kg}$ in Sakiz genotype after baking. It can be safely mentioned that luteolin, rhoifolin, and syringin in the artichoke were shown to be significantly affected by cooking applications. Flavonoids begin thermal degradation during cooking applications, and the losses were noted in studies about vegetables.

\section{CONCLUSION}

In order to improve the functional activities of fruits, vegetables, and meat, the effective cooking method should be examined to keep the total phenolic compounds, antioxidant activities, and amounts of phenolic substances at the highest levels. In the present article, total phenolic compounds and antioxidants of cooked and raw Bayrampasa and Sakiz variety artichokes were determined. Boiling, microwaving, and baking methods were applied to artichokes, and the results were discussed.

TPC, DPPH, and CUPRAC values for the leaves of Bayrampasa and stems of Sakiz artichokes were found to be 686 and 1,579 $\mathrm{mg} \mathrm{GAE} / 100 \mathrm{~g}, 478$ and 1,259 $\mathrm{mg} \mathrm{TE} / 100 \mathrm{~g}$, and 4,875

\section{REFERENCES}

Angelov, G., Georgieva, S., Boyadzhieva, S., and Boyadzhiev, L. (2015). Optimizing the extraction of globe artichoke wastes. Comptes. Rendus de l'Academie Bulg. des Sci. 68, 1235-1240.

Apak, R., Guclu, K., Ozyurek, M., and Karademir, S. E. (2004). Novel total antioxidant capacity index for dietary polyphenols and vitamins $\mathrm{C}$ and $\mathrm{E}$, using their cupric ion reducing capability in the presence of neocuproine: CUPRAC method. J. Agric. Food Chem. 52, 7970-7981. doi: 10.1021/jf048741x

Christaki, E., Bonos, E., and Florou-Paneri, P. (2012). Nutritional and functional properties of Cynara crops (globe artichoke and cardoon) and their potential applications: a review. Int. J. Appl. Sci. 2, 64-70.

Falleh, H., Ksouri, R., Chaieb, K., Karray-Bouraoui, N., Trabelsi, N., Boulaaba, M., et al. (2008). Phenolic composition of Cynara cardunculus L. organs, and their biological activities. C. R. Biol. 331, 372-379. doi: 10.1016/j.crvi.2008.02.008 and 3,575 mg TE/100 g, respectively. An effective increment in the content of TPC, DPPH, and CUPRAC values was obtained for boiling, baking, and microwave cooking applications of both artichokes. It was found that TPC, DPPH, and CUPRAC values rose 2.2, 11, and 43 times after baking of Sakiz. The TPC value of Bayrampasa increased by 11 times after boiling application, whereas DPPH and CUPRAC changed 17 and 6.7 times after microwave cooking, respectively. According to the results, it can be safely stated that chlorogenic acid, cynarine, and cynaroside, which are very important phenolic components for human health, have increased significantly in all cooking types in Sakiz and Bayrampasa artichokes. Chlorogenic acid, cynarine, and cynaroside contents were improved 29, 16.5, and nearly 5 times after baking of Sakiz, whereas they increased by 58, 61, and 16 times after microwave cooking of Bayrampasa, respectively.

It can be safely stated that all cooking methods had a significant effect on the content of total phenolic compounds, antioxidants, and phenolic compounds of Bayrampasa and Sakiz variety artichokes.

\section{DATA AVAILABILITY STATEMENT}

The original contributions presented in the study are included in the article/supplementary material, further inquiries can be directed to the corresponding author/s.

\section{AUTHOR CONTRIBUTIONS}

SK performed experiments, analyzed data, and wrote the paper. DS developed analytical tools, designed experiments, analyzed data, and wrote the paper. All authors contributed to the article and approved the submitted version.

\section{ACKNOWLEDGMENTS}

The financial support for this manuscript was provided by Yalova University Scientific Research Projects Coordination Department (Project no. 2019/DR/0001) and also the Agriculture and Forestry General Directorate of Agricultural Research and Policies R\&D Support Program (Project no: TAGEM/HSGYAD/A/20/A3/P4/1927).
Ferracane, R., Pellegrini, N., Visconti, A., Graziani, G., Chiavaro, E., Miglio, C., et al. (2008). Effects of different cooking methods on antioxidant profile, antioxidant capacity, and physical characteristics of artichoke. J. Agric. Food Chem. 56, 8601-8608. doi: 10.1021/jf80 0408w

Fratianni, T. M., De Palma, M., Pepe, R., and Nazzaro, F. (2007). Polyphenolic composition in different parts of some cultivars of globe artichoke (Cynara cardunculus L. var. Scolymus (L.) Fiori). Food Chem. 104, 1282-1286. doi: 10.1016/j.foodchem.2007.01.044

Jiménez-Monreal, A. M., García-Diz, L., Martínez-Tomé, M., Mariscal, M. M. M. A., and Murcia, M. A. (2009). Influence of cooking methods on antioxidant activity of vegetables. J. Food Sci. 74, H97-H103. doi: 10.1111/j.1750-3841.2009.01091.x

Kayahan, S., and Saloglu, D. (2020). Optimization and kinetic modelling of microwave assisted extraction of phenolic contents and 
antioxidants from Turkish artichoke. CYTA J. Food. 18, 635-643. doi: 10.1080/19476337.2020.1800103

Lattanzio, V., Kroon, P. A., Linsalata, V., and Cardinali, A. (2009). Globe artichoke: a functional food and source of nutraceutical ingredients. J. Funct. Foods 1 , 131-144. doi: 10.1016/j.jff.2009.01.002

Luo, L., Hao, Y., Jia, L., and Zhu, W. (2016). Analysis of chlorogenic acid oxidation pathway in simulated enzymatic honeysuckle browning system by high performance liquid chromatography and mass spectrometrytropical. Trop. J. Pharm. Res. 15, 405-409. doi: 10.4314/tjpr.v1 $5 \mathrm{i} 2.25$

Pandino, G., Lombardo, S., and Mauromicale, G. (2013). Globe artichoke leaves and floral stems as a source of bioactive compounds. Ind. Crops Prod. 44, 44-49. doi: 10.1016/j.indcrop.2012.10.022

Rinaldi, M., Littardi, P., Cavazza, A., Santi, S., Grimaldi, M., Rodolf,i, M., et al. (2020). Effect of different atmospheric and sub atmospheric cooking techniques on qualitative properties and microstructure of artichoke heads. Int. Food Res. J. 137:109679. doi: 10.1016/j.foodres.2020.109679

Stumpf, B., Kunne, M., Ma, L., Xu, M., Yan, F., Piepho, H. P., et al. (2019). Optimization of the extraction procedure for the determination of phenolic acids and flavonoids in the leaf of globe artichoke (Cynara Cardunculus Var. Scolymus L.). J. Pharm. Biomed. Anal. 177:112879. doi: 10.1016/j.jpba.2019.112879
Turkish Standard (1998). Fruit and Vegetable Products-Determination of Dry Matter by Drying Under Low Pressure and Water Content by Azeotropic Distillation. Ankara: Turkish Standards Institue, TS 1129 ISO 1026

Conflict of Interest: The authors declare that the research was conducted in the absence of any commercial or financial relationships that could be construed as a potential conflict of interest.

Publisher's Note: All claims expressed in this article are solely those of the authors and do not necessarily represent those of their affiliated organizations, or those of the publisher, the editors and the reviewers. Any product that may be evaluated in this article, or claim that may be made by its manufacturer, is not guaranteed or endorsed by the publisher.

Copyright (๑) 2021 Kayahan and Saloglu. This is an open-access article distributed under the terms of the Creative Commons Attribution License (CC BY). The use, distribution or reproduction in other forums is permitted, provided the original author(s) and the copyright owner(s) are credited and that the original publication in this journal is cited, in accordance with accepted academic practice. No use, distribution or reproduction is permitted which does not comply with these terms. 